Revista Iberoamericana, Vol. LXIX, Núm. 203, Abril-Junio 2003, 401-415

\title{
LOS ESTUDIOS CULTURALES: GEOPOLÍTICA DEL CONOCIMIENTO Y EXIGENCIAS/NECESIDADES INSTITUCIONALES
}

\author{
POR \\ Walter D. Mignolo \\ Duke University
}

1. INTRODUCCIÓN: UN MAPA DE PROBLEMAS

Tres meses antes de la reunión de LASA (Washington, 6 al 9 de septiembre de 2001), esto es, hacia el 10 de junio de 2001, la Universidad Andina de Quito organizó una conferencia internacional sobre Estudios Culturales con motivo del comienzo de un programa de pos-grado en este ámbito disciplinar o interdisciplinar. Varios de los participantes en los tres paneles de LASA que se recogen en esta publicación estuvieron en Quito. ${ }^{1}$ En Quito, Santiago-Castro Gómez abrió la conferencia con una anatomía de las críticas que la derecha intelectual y universitaria hace a los Estudios Culturales. Su presentación mostró los temores y las desconfianzas que los Estudios Culturales despiertan en los sectores más conservadores de la academia y de la derecha intelectual, incluido el periodismo. Castro-Gómez se ocupó del libro de Carlos Reynoso titulado Apogeo y decadencia de los estudios culturales. Una visión antropológica, publicado en julio del 2000 por la editorial Gedisa de Barcelona. Para Castro-Gómez este libro “constituye, sin lugar a dudas, un desafío y un estímulo para la práctica teórica de los estudios culturales en América Latina. ${ }^{2}$ El libro de Reynoso subraya otro eje de las polémicas y los resquemores que despiertan los estudios culturales. Un eje es el debate en Estados Unidos que aparece en publicaciones como Social Text o The Chronicle of Higher Education, y otro es el debate en América Latina. Al mismo tiempo, el debate en América Latina se vuelve sobre el debate en Estados Unidos en la medida en que la cuestión de los estudios culturales se entremezcla con los estudios de áreas. Esto es, tenemos por un lado el debate sobre los estudios culturales "en general” y, por el otro, los estudios culturales y los estudios deáreas. Es decir, estudios latinoamericanos y estudios culturales latinoamericanos. En Estados Unidos, claro. Para aclarar un poco estas difusas configuraciones, distingamos

\footnotetext{
${ }^{1}$ Entre los que estuvieron en Quito y en estas reuniones de LASA están Mabel Moraña, John Beverley, Daniel Mato y yo. Entre los que estuvieron en Quito pero no en los paneles de LASA, aunque sus trabajos son relevantes para el tema en cuestión, están Santiago Castro-Gómez, Catherine Walsh, Javier Sanjinés, Oscar Guardiola, Fernando Coronil y Edgardo Lander.

${ }^{2}$ La ponencia se tituló "Apogeo y decadencia de la teoría tradicional. Una visión desde los intersticios".
} 
entre la dimensión institucional de los estudios culturales y los proyectos intelectuales que se cobijan bajo el nombre (o que usan ese nombre para cobijarse).

Sobre el aspecto institucional la pregunta es: ¿qué papel tienen los estudios culturales en la perspectiva de los administradores de las mayores universidades en Estados Unidos? Aquí sólo menciono Estados Unidos porque desconozco este aspecto, del que voy a hablar en este párrafo, en América Latina, aunque sé, por ejemplo, que la Universidad Andina Simón Bolívar, en Quito, está lanzando un programa de estudios culturales. Desde el punto de vista institucional, las administraciones de las universidades estadounidenses tienen dos razones, al menos, para apoyar los estudios culturales. Una es económica. Los estudios culturales presuponen interdisciplina, e interdisciplinar presupone que una persona puede ocupar puestos en dos departamentos. La otra es política. Los investigadores y profesores en las universidades caracterizadas como "research universities" de tendencia interdisciplinaria contribuyen a abrir y a des-osificar departamentos y programas osificados por la permanencia de investigadores y profesores más apegados a las normas disciplinarias que a las aventuras interdisciplinarias. Esto es, institucionalmente los estudios culturales permiten construir un argumento de reforma de las disciplinas y de apertura interdisciplinaria.

$\mathrm{Al}$ aspecto institucional (e.g., la creación de departamentos o de programas de Estudios Culturales) y el administrativo (e.g., las repercusiones en el presupuesto, en la política administrativa universitaria, en los cambios en las disciplinas y en las estructuras de poder de los departamentos universitarios), hay que agregar el aspecto intelectual. Es decir, ¿cuáles son los proyectos intelectuales que necesitan de la institucionalización de los Estudios Culturales y de la interdisciplinaridad y que no podrían llevarse adelante en los departamentos en los cuales la disciplina tiene prioridad sobre la interdisciplina? La formulación de esta pregunta es un tanto rígida. Ella presupone que, por un lado, están los departamentos puramente disciplinares (sociología, filosofía, literatura, historia, biología, informática, etc.) y por otro los estudios culturales abiertamente interdisciplinarios. Si las cosas fueran así, no habría cabida en ninguno de los departamentos organizados en torno a una sola disciplina para amparar proyectos de investigaciones y planes de enseñanza (currículo) que no respondieran a los cánones de las disciplinas. Sabemos que esto no es así y que las cosas no dependen tanto de las disciplinas como de las personas que actúan (investigando, enseñando, participando en organizaciones disciplinarias nacionales e internacionales, asociaciones de sociología, filosofía, historia, literatura, historia del arte, del estudio de las religiones, etc.). De tal manera que es imperativo separar las necesidades institucionales y administrativas, por un lado, de las necesidades (y deseos) intelectuales, por el otro. O, mejor aún, es imperativo separar las políticas institucionales y administrativas de las políticas y la ética de la investigación y de la enseñanza. Los millones de dólares que fluyen desde fundaciones a universidades en Estados Unidos para “repensar esto o aquello", para mejorar el entrenamiento de estudiantes de pos y pre-grado, no necesariamente mejoran la situación si no hay proyectos intelectuales innovadores. Más dinero en la institución es un fenómeno grato pero que de ninguna manera asegura proyectos intelectuales críticos e innovadores. Ese dinero puede, en verdad, contribuir a fomentar la razón instrumental (eficiencia en la producción y en la administración de bienes y servicios, incluida la información entendida como educación) y la razón estratégica (cómo vencer al “enemigo”, cómo sacar ventaja de la situación X 
o Z, cómo acrecentar el espacio en el ejercicio del poder, etc.) en vez de la razón crítica (qué tipo de conocimiento y comprensión tiene urgencia social o está impulsada por el deseo; desde qué perspectiva — cuáles son los principios asumidos que dirigen la producción y transformación de conocimientos- y qué métodos y teorías se disponen o hay que inventar). Por último, con qué fines se genera tal conocimiento o comprensión. Demás está decir que la razón crítica sería el horizonte último de las Humanidades entendidas en un sentido amplio. Claro está, no en la dimensión institucional en la cual se agrupan los humanistas, sino en la intelectual, ética y política en la que todo conocimiento y comprensión (desde la medicina a los negocios, desde la ingeniería al derecho, desde las ciencias naturales a las sociales) son -y no pueden ser de otra maneraciencias humanas. Sólo los seres humanos pueden producir, almacenar, olvidar, transformar conocimiento y comprensión. No estoy sugiriendo, ni tampoco voy a sugerir, que el mapa que estoy diseñando es el de los Estudios Culturales. No, todo lo contrario. Estoy sugiriendo que, en el terreno intelectual, hay tareas mucho más urgentes que la de discutir si vale o no la pena defender o denigrar a los Estudios Culturales y esa tarea es la de vigorizar la razón crítica en las Humanidades que perdieron terreno por el avance de la razón instrumental y la razón estratégica, cada vez más omnipresentes en las universidades de Estados Unidos, Europa y la zona colonial de influencia en la cual se crearon universidades y centros de estudio y de investigación desde el siglo xvi. La Universidad de México, por ejemplo, creada en 1553 y la de Harvard, en 1634.

En fin, mi argumento hasta aquí es que no hay en realidad una relación uno-a-uno entre un cierto orden institucional, digamos, los Estudios Culturales y lo que se hace bajo ese nombre o rubro. Y también a la inversa es válido. Iniciativas y proyectos intelectuales que no cuadran en el marco de las disciplinas canónicas no tienen necesariamente que justificarse y buscar un techo en los Estudios Culturales. Los Estudios Culturales son o pueden ser un espacio institucional de conveniencia, y nada más. No obstante, es muy importante poder contar con este lugar recordando que es un espacio institucional de conveniencia y nada más. Esta separación es importante por muchas razones, como veremos. Una de las razones fundamentales es la de clarificar los criterios bajo los cuales un rubro institucional, Estudios Culturales, se introduce en las universidades de Ecuador, Colombia o Argentina — por ejemplo— sin necesariamente introducir también los proyectos intelectuales que en Inglaterra o en Estados Unidos se cobijaron bajo el mismo rubro. Así las cosas, podríamos pensar que si los estudios culturales británicos se distinguieron por sus contribuciones a la comprensión de los problemas de la juventud y de los medios de comunicación, los problemas de la juventud y de los medios de comunicación no deberían imponerse en Ecuador o en Colombia porque estos son proyectos de los estudios culturales tal como se lo definió en Inglaterra, sino porque en Ecuador y Colombia hay necesidad de tales estudios. Esto es, la creación de un departamento o programa de estudios culturales en Chile, Argentina o Ecuador no se justificaría si sólo fuera para modernizar la universidad. Es decir, se justificaría, pero la justificación sería institucional y administrativa, no intelectual. Sería un argumento de la razón estratégica. La justificación intelectual (la de la razón crítica) tiene otra lógica. Primero importa saber cuáles son los problemas que requieren atención en los Andes, en Quito, en Colombia o en Bolivia o en Estados Unidos. Esta es una decisión que deben 
hacer aquellos que están involucrados en el proyecto. Además, habría la necesidad de crear un espacio institucional donde se pudieran realizar proyectos intelectuales, política y éticamente sustentados, que no se podrían realizar en las estructuras universitarias actuales. Desde la perspectiva ética y política del conocimiento, lo fundamental son los problemas y las preguntas que motivan la investigación (razón crítica). Desde la perspectiva política de la institución, lo fundamental es crear espacios que permitan transformaciones institucionales (como las que mencioné en el caso de Estados Unidos) y que en América Latina podrían justificarse, institucionalmente, como modernización académica (razón estratégica) y para la preparación de personas técnicamente capacitadas para obtener una producción eficiente y de buena calidad (razón instrumental).

Hay todavía un último problema, entre aquellos de los que me ocuparé aquí, para poner sobre la mesa. Este problema se deriva de la conceptualización de los Estudios Culturales, sin más, y de los Estudios Culturales entrelazados con la herencia de los Estudios de Áreas. Si bien los Estudios de Áreas son un asunto de la universidad en Estados Unidos, estos no pueden ignorarse en América Latina (o en África y Asia) puesto que estas "áreas" fueron convertidas en objetos académicos de las universidades estadounidenses. Por esta razón tenemos hoy un abanico geo-político tal que abarca Estudios Culturales Latinoamericanos, Estudios Culturales Africanos, Asiáticos, Chicanos/ as, etc. De tal manera que pareciera que los Estudios Culturales están repitiendo una estructura disciplinaria que se consolidó durante la guerra fría y que se definió por un tipo particular de relación entre las disciplinas que producen conocimiento (knowledge) y los contenidos de ese conocimiento que fueron las "áreas" en que se dividió el mundo que debía conocerse (the known and the knowable). Durante el período de la guerra fría teníamos, por un lado, las disciplinas (sociología, historia, antropología) y por el otro las áreas (América Latina, África, Asia, Asia Central, Caribe, etc.).

De tal modo que, teniendo en cuenta este escenario, habría una diferencia interesante para desenredar cuando se compara la situación hoy, en los Estudios Culturales, y la situación ayer, en la tensión entre disciplinas y áreas. Mientras que ayer, es decir, durante la guerra fría, las áreas convocaban un número distinto de disciplinas (América Latina era estudiada por economistas, sociólogos, historiadores, antropólogos) para estudiarlas y cada una de estas disciplinas contribuía a explorar un aspecto de la complejidad, aquel aspecto que correspondía a la disciplina, los Estudios Culturales Latinoamericanos (o Asiáticos o Africanos) no tienen hoy un perfil definido. Por un lado, los Estudios Culturales provienen, en general, de los estudios literarios y de la apertura interdisciplinaria del pos-estructuralismo. Por su parte, los Estudios Culturales Latinoamericanos, en Estados Unidos, ofrecen una alternativa a los estudios de literatura latinoamericana, por un lado y a los estudios latinoamericanos de áreas por el otro. La primera esfera, literatura, está asignada a las humanidades mientras que la segunda a las ciencias sociales. ¿Cuáles son las diferencias, en última instancia, entre los Estudios Culturales Latinoamericanos y los Estudios Latinoamericanos tal como se los institucionalizó durante la guerra fría a través de LASA (Latin American Studies Association)? Sin duda que ésta es una pregunta que no puedo responder aquí en detalle. Me conformo con sugerir lo siguiente: mientras que los Estudios Latinoamericanos, en el sentido de Latin American Studies y de la correspondiente asociación, Latin American Studies Association (LASA) fueron - y 
todavía son- básicamente un asunto de las ciencias sociales, los Estudios Culturales Latinoamericanos emergieron como un asunto, fundamentalmente, de las humanidades. Además, si “América Latina” se convirtió en el objeto de "estudio" de los "Estudios" Culturales, entonces la política y la ética de la investigación no pueden ser las mismas cuando estos “estudios” se practican en Estados Unidos y en América Latina. Esto es, no pueden ser lo mismo desde la perspectiva geopolítica del conocimiento. En cambio, si pensamos que los Estudios Culturales son geopolíticamente neutros y que es lo mismo practicar Estudios Culturales en Londres, en Pittsburgh o en Quito (puesto que la epistemología es neutra y ahistórica, no tiene ni sexo ni color), entonces los Estudios Culturales son neutros con respecto a su ubicación geohistórica e institucional. Siguiendo este argumento resultaría entonces que aquello que se necesita saber, en Estados Unidos, sobre América Latina no sería distinto a lo que se necesita saber en América Latina. Por esta razón, se teme que las agendas intelectuales de Estados Unidos sean impuestas en América Latina puesto que aquellos que expresan este temor saben que es muy difícil que las agendas intelectuales de América Latina se impongan en la academia estadounidense. En un mundo ideal donde no hubieran distinciones geopolíticas organizadas por la colonialidad del poder y la diferencia colonial, los "Estudios Culturales Latinoamericanos" en América Latina serían equivalentes a los “Estudios Culturales (Anglo) Americanos” en Estados Unidos. De esta manera, todos los practicantes de uno y de otro campo, felices y simétricos, cambiarían información en bien de la acumulación y el progreso del saber. Pero al parecer, la realidad no es así. ${ }^{3}$

Este tipo de argumento requiere explorar una serie de preguntas sobre el orden institucional y la geopolítica del conocimiento, que paso a enumerar:

1) ¿Cuáles son las necesidades institucionales, administrativas e intelectuales que satisfacen la creación de un departamento o programa de Estudios Culturales en Quito, Bogotá, Buenos Aires, etc.?

2) ¿Cuáles son las necesidades institucionales, administrativas e intelectuales que satisfacen la creación de un departamento o programa de Estudios Culturales Latinoamericanos en Estados Unidos?

3) Mientras que en Estados Unidos el adjetivo "Latinoamericano" especifica América Latina como objeto, la práctica de los Estudios Culturales en América Latina no tiene necesariamente que ser especificada de ese modo. Los Estudios Culturales, en Quito, Bogotá o Buenos Aires tienen la libertad y el privilegio de hacer de la globalización el objeto de estudio y no de reproducir, en América Latina, las agendas intelectuales de los Latin American Studies o de los Latin American Cultural Studies, si los hubiera. El Plan Colombia, por ejemplo, no es un asunto que tiene que ver con la historia de América Latina solamente. Es un problema de la globalización. Así como los Estudios Culturales en Quito, Bogotá, Buenos Aires, etc. tienen la libertad y el privilegio de estudiar el mundo, si se especifican como latinoamericanos corren el peligro de transformarse en un "token" cultural, semejante al caso analizado hace algunos años por George Yúdice en el que un museo de Houston esperaba que los artistas latinoamericanos invitados "pintaran"

\footnotetext{
${ }^{3}$ Ver Daniel Mato 2001.
} 
América Latina y no que tuvieran el atrevimiento de producir y pensar el arte a través de su pintura. Uno tiene la sensación, por ejemplo, de que en las universidades de Estados Unidos se esperaría que “los latinoamericanos se ocupen de asuntos latinoamericanos” y no de pensar el mundo a través de América Latina, al igual que lo hacen los pensadores europeos o estadounidenses. Esto es, la globalización se estudia en Alemania, Inglaterra, Francia, Estados Unidos o Japón, pero en América Latina sólo se estudia América Latina. Sin duda que los trabajos de Néstor García Canclini, Jesús Martín Barbero, Renato Ortiz, Octavio Ianni, José Joaquín Brunner, etc. se conocen mayormente en América Latina. Estos estudios no entran en el debate internacional junto a Anthony Giddens, Saskia Sassen, Ulrich Beck o Pierre Bourdieu. Y no estoy aquí hablando o esperando "reconocimiento intelectual" sino de estructuras institucionales de saber y de poder, aún mejor, de la persistencia de la colonialidad del poder aquí manifiesta en el orden epistémico e institucional universitario. Por esta razón la geopolítica del conocimiento es una cuestión que debe explorarse con prioridad a la institucionalización de los Estudios Culturales, o de cualquier otro tipo de "estudios".

\section{Algunas historias locales y algunas agendas intelectuales}

Exploremos algunos ejemplos donde podamos ver la interrelación entre proyectos intelectuales y formalización institucional. Veamos, a vuelo de pájaro, qué ocurría en Inglaterra cuando se institucionalizaron los Estudios Culturales (más específicamente, el Centro de Estudios Culturales Contemporáneos) en los tempranos años $60 .{ }^{4}$ Veamos qué ocurría en América Latina en esos momentos. Y veamos también el panorama de la misma década en Estados Unidos. El contraste y las relaciones entre estos tres momentos nos darán una perspectiva histórica para comprender las relaciones entre las políticas institucionales y administrativas, por un lado, y los proyectos intelectuales por el otro. Estas breves viñetas nos ayudarán además a comprender qué es lo que está en juego, a principios del siglo XXI, en la defensa o en la promoción de los Estudios Culturales. Y a pensar también cuáles serían los proyectos intelectuales en distintas historias locales, digamos en América Latina, en Inglaterra y en Estados Unidos. Podría también mencionar lugares del Pacífico, como Taiwán o Japón o Hong-Kong, pero esto complicaría un tanto las cosas. Sin embargo, haré algunas referencias, más abajo, para tener una perspectiva geo-política, y no universalista, de los estudios culturales. ${ }^{5}$

\footnotetext{
${ }^{4}$ Para un panorama de la emergencia de los Estudios Culturales en Inglaterra ver Dworkin 1997. Para el contexto histórico, en Estados Unidos, que provocó la emergencia de Women Studies, Ethnic Studies, Chicano y luego Latino Studies, Afro-American Studies, etc., ver Frankenberg y Mati (1993). Las exigencias que se derivan del movimiento de los derechos civiles, más la creciente inmigración desde el tercer mundo, particularmente de América Latina, crearon las condiciones para la emergencia de estudios de género y de etnicidad, de sexualidad y de racismo. Los Estudios Culturales llegaron tardíamente a Estados Unidos y por otras exigencias que tienen que ver más con la relación entre Estados Unidos y Europa (Francia y el pos-estructuralismo; Inglaterra y los Estudios Culturales ligado a la particular "tradición" marxista en Estados Unidos; Alemania y la segunda ola de la Escuela de Frankfurt-Habermas, etc.).
} 
En fin, no creo que tenga ningún sentido hoy, sobre todo en América Latina pero también para los practicantes de los estudios latinoamericanos (culturales o no) en Estados Unidos, involucrarse en proyectos institucionales que no comiencen por una reflexión crítica sobre las genealogías de los proyectos intelectuales que son los que verdaderamente cuentan. El nombre, la formalización institucional corresponde a otro tipo de política, la política precisamente institucional, no a la política intelectual. No hay una relación uno a uno entre la institucionalización de los estudios culturales y los proyectos intelectuales que se pueden albergar bajo su techo. Creer que se trata de lo contrario es abrir las puertas a quienes se interesan más por el poder que por el pensamiento y que, en consecuencia, intentan imponer una agenda de pensamiento y controlar su lugar institucional, se llamen estudios culturales u otra cosa. Por eso, repito, es necesario separar el nivel institucional de los proyectos intelectuales. Los proyectos intelectuales no necesitan de los estudios culturales puesto que, como proyectos intelectuales, tienen posibilidad de llevarse adelante por otros canales. En cambio, la institucionalización de los estudios culturales necesita de proyectos intelectuales puesto que de otra manera, ¿qué es lo que se institucionalizaría? Un lugar vacío que espera, como en el caso de Godot o del actor pinrandeliano, a un autor.

2.1. En Inglaterra, la creación del CCCS (Centre for Contemporary Cultural Studies) en Birmingham, en 1964, tiene una historia — icomo es de suponer! — tanto intelectual como institucional: la historia intelectual en las condiciones de la Inglaterra de pos-guerra (ver nota 4). Por un lado, la insatisfacción de los jóvenes intelectuales marxistas (Raymond Williams, J. P. Thompson, Richard Hoggart) con las formas canonizadas del marxismo, esto es, del leninismo-stalinismo. La falta de atención que los marxistas le habían prestado a la cultura, hasta esos momentos, así como la apropiación del tema de la “cultura” por críticos e intelectuales de derecha como F. R. Leavis, condujo al encuentro de la Nueva Izquierda con la cultura. Un tema que no se menciona en los análisis de este momento, es la descolonización de India, en 1947, y la eminente pérdida de las posesiones inglesas en el futuro próximo. Pero esta ausencia no debe sorprender a nadie, lo mismo ocurrió en Francia. La voluptuosidad del estructuralismo y del pos-estructuralismo fueron asuntos internos de Europa, de espaldas a las colonias, a la descolonización de Argelia en 1963 y a la historia que siguió en el proceso descolonizador. Raymond Williams no

\footnotetext{
${ }^{5}$ Un caso interesante para ilustrar este argumento es el del taiwanés Kuan-Hsing Chen. Estudió con Stuart Hall y editó con David Morley uno de los mejores volúmenes sobre la obra de Hall (Morley y Chen). Sin embargo, ni bien regresó a Taiwán planteó el problema de la diferencia epistémica colonial al contrastar la práctica de los estudios culturales en Taiwán, y en el Este Asiático, y en Inglaterra y el Atlántico Norte ( Chen). No tardó tampoco en crear su propia revista, Inter-Asia Cultural Studies, la cual comenzó con un volumen titulado “Problematizing ‘Asia”” (vol. 1.12000 ). Por todo lo que he estado diciendo sobre América Latina, no es de sorprender que Kuan-Hsing, cuyo proyecto intelectual me parece estupendo, comience por problematizar la idea de "Asia” y no la idea de "Estudios Culturales". Problematizar Asia, en el momento de la rearticulación de la economía global y sus consecuencias culturales, políticas, éticas y epistémicas es un proyecto intelectual radical. En cambio, problematizar "Estudios Culturales” es una cuestión de política institucional, de razón estratégica más que de razón crítica.
} 
incorporó para nada “colonización” (y menos aún “descolonización”) ni “raza” en su colección de palabras claves.

Sin embargo, la confluencia de la nueva izquierda con los estudios culturales renovó el pensamiento marxista en los años 60 en Inglaterra. Así, es justo afirmar que la escuela británica de los estudios culturales: "was an unprecedented international boom” y que los estudios culturales británicos (y subrayo británicos en comparación con latinoamericanos)

\begin{abstract}
has advanced critical understanding of the media, youth subcultures, literary production, the contemporary working class, the cultural construction of race and gender, popular culture, and the nature of ideology. It is distinguished by its simultaneous respect for the potentially subversive culture of dominated and marginalized classes and groups and an acute awareness of the ideological forces in society containing them. Interdisciplinary and theoretically eclectic, cultural studies has supplanted the traditional dichotomy between high and low culture, so prevalent in discussions about the mass media, with an enlarged concept of the cultural terrain. (Dworkin 1997 2; ver la nota 4)
\end{abstract}

En suma, el Centre for Contemporary Cultural Studies surgió en el proceso de renovación del pensamiento marxista en una Inglaterra de posguerra y en el momento en que el Imperio Británico se encontraba en una situación semejante a la que se encontró España a principios del siglo XIX, como consecuencia de las independencias en América Latina. ¡Pero el marxismo no existía entonces! El CCCS tuvo su primer hogar en el English Department de la Universidad de Birmingham, aunque el departamento rehusó apoyarlo económicamente. El apoyo económico vino en parte de los propios miembros y particularmente del esfuerzo inicial de Richard Hoggart.

La trayectoria de la reflexión crítica marxista, en Inglaterra, se toparía hacia finales de los años 60 con el althuserianismo y con el auge del pensamiento pos-estructuralista. Esta historia interesa en la medida en que se encuentra viviendo en Inglaterra, en esos años, un historiador de origen hindú llamado Ranajit Guha. Si bien Guha, en tanto marxista, no puede ignorar los trabajos de E. P. Thompson, Richard Hoggart, Raymond Williams y Stuart Hall, su proyecto no es exactamente el de estos pensadores. A Guha no le interesa la renovación del pensamiento marxista en Inglaterra, sino la situación política y la escritura de la historia en la India pos-1947. Guha tiene preocupaciones similares a las del grupo que fundará el Centro de Estudios Culturales Contemporáneos pero, al mismo tiempo, tiene que cocinar otra sopa, la suya, la de la descolonización de India más que la de la desimperialización de Inglaterra. Guha fundó su grupo de trabajo hacia 1973 y el primer volumen de South Asian Subaltern Studies se publicó en 1982.

Es útil recordar aquí los debates intelectuales en América Latina a partir de la década del 50 y del 60, con el informe de la CEPAL y la emergencia de la teoría de la dependencia. El límite de espacio no permite recordarlos en detalle, sólo invocarlos (ver Grosfoguel 2000). Es importante también recordar aquí lo que ocurrió en Estados Unidos entre el 50 y el 70. Subrayemos dos acontecimientos relevantes para esta argumentación. El movimiento de Civil Rights y la emergencia del World-System Analysis. El posestructuralismo había "llegado" a principios de los 70. Y Edward Said había abierto otras puertas con Orientalism, publicado en 1978. En ese clima "llegaron" los estudios 
culturales a Estados Unidos, a comienzos de los 80, desde Inglaterra. Cary Grossberg y Larry Nelson organizaron una conferencia, en Urbana, Illinois, titulada Marxism and the Interpretation of Culture. Los estudios culturales entraron en Estados Unidos reclamando la necesidad de articular las ciencias sociales y las humanidades, porque los organizadores del encuentro y editores del libro bajo el mismo título (1988), sostenían que el marxismo,

... has long been at least implicitly involved in breaking down the barriers between these domains, making each necessity a site of interpretative activity — by politicizing interpretative and cultural practices, by looking at the economic determinations of cultural production, by radically historicizing our understanding of signifying practicesfrom political discourse to art, from beliefs to social practices, from the discourse of psychology to the discourse of economics — and, of course, by continuing to revise and enlarge a body of theory with multidisciplinary implications. (Dworkin 1)

Al revisar las historias políticas e intelectuales, paralelas y entrecruzadas, de Inglaterra, América Latina y Estados Unidos (dos países y un sub-continente; dos países de lengua inglesa y un sub-continente donde las lenguas mayoritarias son el español y el portugués), se me hace patente que falta, tanto en América Latina como en Estados Unidos, una reflexión seria sobre lo que significan "los estudios culturales latinoamericanos" en Estados Unidos y los “estudios culturales” en América Latina. Digo “estudios culturales en América Latina” puesto que, por un lado, Grossberg y Nelson no hablaron de "estudios culturales americanos", sino de "estudios culturales" a secas. Por lo tanto, los "estudios culturales latinoamericanos en Latinoamérica” darían lugar a una actitud inicial de dependencia con los “estudios culturales latinoamericanos en Estados Unidos”.

\section{Algunas Reflexiones generales}

¿Para qué y por qué contar la historia que acabo de contar? Al reflexionar sobre ella vemos que, en Inglaterra, hubo una relación orgánica entre un proyecto intelectual de renovación del marxismo y la creación del Centro de Estudios Culturales Contemporáneos. En cambio, en la conferencia de Illinois, y en la subsecuente publicación del libro, Marxism and the Interpretation of Culture, el proyecto no estuvo sustentado por exigencias de la historia local, de Estados Unidos, como lo fue en el caso de Inglaterra, sino más bien motivado por el "efecto estudios culturales" que se había generado en Inglaterra hacía unos veinte años. Lo mismo ocurrió, en Estados Unidos también, con el surgimiento de los Estudios Subalternos Latinoamericanos (ver Rodríguez). Mientras que en la India, después de la descolonización de 1947, fue la historia misma de India la que motivó la creación del Grupo Surasiático de Estudios Subalternos en Inglaterra, la creación de los Estudios Subalternos Latinoamericanos fue consecuencia del "efecto estudios subalternos" en la India como respuesta a la crisis de Nicaragua y a la crisis de la izquierda al terminar la guerra fría. En fin, los Estudios Subalternos Surasiáticos surgieron en la segunda mitad de la guerra fría, mientras que los Estudios Culturales en Birmingham, al comienzo de ella. La “adaptación” de los estudios subalternos a los estudios latinoamericanos, entre los estudios literarios y los estudios de área, ocurrió cuando la guerra fría ya había terminado. 
Mientras que los dos procesos, estudios culturales y estudios subalternos en Inglaterra, fueron el resultado de "exigencias históricas e intelectuales", en Estados Unidos fueron más bien el resultado de "la extensión de un modelo que tuvo éxito” y del orgullo del latinoamericanista en universidades estadounidenses de adoptar esta vez — como alguien dijo - un modelo del tercer mundo en vez de adoptar un modelo del primer mundo. No dudo de las buenas intenciones de quienes siguieron este segundo camino, pero tampoco quiero pasar por alto las diferencias entre las exigencias institucionales y las necesidades intelectuales. En cambio, hubo en los estudios étnico-raciales de diverso tipo, en Estados Unidos, como también en los estudios de género y sexualidad, relacionados con el movimiento de reclamo de derechos civiles, un arraigo y una estrecha relación entre proyecto intelectual, primero, y solución institucional, segundo, que asemejan estos procesos a lo que ocurrió en Inglaterra. Esto es, mientras que la emergencia de los estudios culturales y subalternos en Inglaterra e Inglaterra-India, y los estudios de raza y género en Estados Unidos surgieron de una relación encarnada entre necesidades intelectuales y exigencias institucionales, los estudios culturales y subalternos en Estados Unidos siguieron un proceso inverso: nacieron de exigencias institucionales, y luego se los trató de llenar con proyectos intelectuales.

En cuanto a América Latina, los proyectos encarnados son los que he mencionado. Teoría de la dependencia, filosofía de la liberación, colonialismo interno en la década de los 60 como en su continuidad actual traducida a colonialidad del poder (Quijano), transmodernidad y crítica al Eurocentrismo (Dussel), colonialismo interno, alternativas a la modernidad epistémica, estudios de género y raza (Rivera Cusicanqui, Rossana Barragán), geo-política del conocimiento (Mignolo, Palermo, Walsh, Castro-Gómez, Guardiola, Lander), colonialidad del saber (Lander, Quijano, Mignolo), geopolítica del conocimiento, colonialidad y migraciones (Grosfoguel), modernidades subalternas y críticas al globalcentrismo (Coronil, Quijano, Dussel), etc. Estos proyectos, además, están en diálogo con los proyectos en marcha en Estados Unidos en algunos sectores de los estudios chicanos (Saldívar). ${ }^{6}$ Pero quizás lo que más importa es que en todos estos proyectos el horizonte último no es el que prometen las modernidades alternativas sino, como lo argumenta Escobar, el de las posibilidades de alternativas a la modernidad en

\footnotetext{
${ }^{6}$ Existe ya una amplia bibliografía de un proyecto intelectual en el que se entrecruzan instituciones e intelectuales en América Latina y Estados Unidos en torno a cuestiones de colonialidad y economía, globalización, eurocentrismo, racismo, etc. Entre estas publicaciones hay que mencionar, primero, los trabajos publicados desde finales de los 80 y principios de los 90 en Anuario Mariateguiano, dirigido por Aníbal Quijano y Antonio Melis; varias publicaciones de Enrique Dussel, pero fundamentalmente el volumen III de sus tres volúmenes sobre Marx (El último Marx (1863-1882) y la liberación latinoamericana. México: Siglo XXI, 1990); la sección especial de Comentario Internacional dedicado a Geopolíticas del conocimiento editado por Catherine Walsh en 2001 (http://www.uasb.edu.ec/public/pub/comentario2.htm); los libros editados por Edgardo Lander y publicados por FACES-USV-UNESCO (La colonialidad del saber. Eurocentrismo y ciencias sociales, 2000) (http://www.clacso.org/libros/lander/llander.html); el libro editado por Santiago Castro-Gómez (La reestructuración de las ciencias sociales en América Latina. Bogotá: Instituto Pensar, 2000); el libro editado por Walter Mignolo (Capitalismo y geopolítica del conocimiento: el eurocentrismo y la filosofía de la liberación en el debate intelectual contemporáneo.
} 
la que están ya involucrados varios movimientos sociales y también proyectos intelectuales concurrentes (Escobar “Globalization, Modernity and Development”, presentación oral en Duke University, 2 de octubre de 2001 dentro del seminario "Globalization and Democracy”). Todos fueron, y son, proyectos intelectuales que no tienen una cara institucional. Los estudios poscoloniales y los estudios culturales no fueron tomados con entusiasmo en primera instancia por resultar ajenos al vocabulario y a la genealogía de estos proyectos. Sólo en Bolivia la expresión “poscolonialismo” y “estudios subalternos” ingresó, pero ingresó de una manera natural. Esto es, ingresó como diálogo con algo que ya se estaba haciendo, la crítica al racismo, al colonialismo, a la modernidad y al desarrollo (Rivera Cusicanqui, Barragán).

Por otra parte, el concepto de “estudios culturales” funcionó, hasta ahora, más como un proyecto intelectual que como un lugar institucional. Los estudios culturales estuvieron y siguen estando asociados a los nombres de Néstor García-Canclini y Jesús Martín Barbero. Ambos tienen en común la crítica a la modernidad focalizada en los medios de comunicación. Ahora bien, es importante subrayar que en estos proyectos intelectuales la expresión "estudios culturales" está ligada a la crítica a la modernidad y no a la renovación crítica del marxismo, si bien ni García-Canclini ni Martín Barbero son antimarxistas. Además, hay otra línea de exploración de este proyecto, la globalización, que une a Barbero y Canclini con Renato Ortiz, por ejemplo, en Brasil, y con José Joaquín Brunner, en Chile (sobre este asunto ver Mignolo, “Capitalism...”).

Quedan, para finalizar, un par de cuestiones. Una de ellas es institucional y la otra intelectual epistémica. Institucionalmente, algunos de los asuntos que siguen en pie son, por ejemplo, ¿de qué manera plantearse la creación de un departamento o programa universitario de Estudios Culturales en Quito o en Bogotá? Y ¿de qué manera hacerlo en Estados Unidos? En Estados Unidos se da una distinción de hecho entre Estudios Culturales, sin más, y Estudios Culturales Latinoamericanos, por ejemplo. Los primeros pueden ocuparse de cualquier asunto, incluidos los asuntos latinoamericanos, mientras que los segundos sólo pueden ocuparse de asuntos latinoamericanos. Los primeros están institucionalmente localizados en departamentos de literaturas comparadas o al menos siguen su modelo y en ellos hay pocos investigadores y profesores que se ocupen de América Latina. Los segundos están en general ubicados en institutos de Estudios Latinoamericanos y en ellos hay pocos investigadores y profesores que se ocupen de otra cosa que no sea América Latina.

Buenos Aires: Editorial del Signo, 2001); y varios trabajos importantes de Silvia Rivera Cusicanqui no recogidos en ningún volumen. Para un resumen de ellos ver Walter D. Mignolo. "Descolonización epistémica y ética: la contribución de Xavier Albó y Silvia Rivera Cusicanqui a la reestructuración de las ciencias sociales desde los Andes”. Revista Venezolana de Economía y Ciencias Sociales 7.3 (2001): 85-112; el volumen 1.3 (2000) de Nepantla: Views from South, que recoge trabajos de Dussel, Castro-Gómez, Lander y Quijano, además de los artículos recogidos en La colonialidad del saber y Teorías sin disciplina. Fernando Coronil publicó recientemente otro artículo fundamental para el tipo de reflexiones que organiza esta nota al pie: “Toward a Critique of Globalcentrism” que fue publicado en Public Culture 12.2 (2000), en el "dossier” preparado por Freya Schiwy y Michael Ennis. "Geopolitics of Knowledge: Knowledge and the Known” con artículos de Catehrine Walsh, Oscar Guardiola y Javier Sanjinés en Nepantla. Views from South 2001 2-3. 
¿Cuáles son los escenarios posibles en la creación de programas o departamentos de Estudios Culturales en América Latina? En primer lugar, los estudios culturales en América Latina no tienen porqué ser de "estudios latinoamericanos”. Recordemos que los estudios "latinoamericanos" son la consecuencia de los estudios de áreas y en los estudios de áreas América Latina, y otros lugares del Tercer Mundo, fueron el objeto de estudio mientras que las disciplinas que lo estudiaban estaban institucionalmente localizadas en el Primer Mundo. Por otra parte, los Estudios Culturales en América Latina no tienen por qué definirse necesariamente como "latinoamericanos" para analizar asuntos y problemas que atañen a los habitantes de la región. Además desde Estados Unidos, América Latina es una región recortada sobre sí misma. En cambio, desde América Latina, el problema no está tanto en sí misma como en su lugar en la modernidad y la globalización. Como ya lo muestran los trabajos de García-Canclini, Martín Barbero, Renato Ortiz, Brunner, Silvia Rivera, Rosana Barragán, los proyectos intelectuales que se generan y se llevan adelante en América Latina tienen como objeto el mundo, la modernidad, la globalización. De lo contrario, si los Estudios Culturales en América Latina se ocuparan sólo de América Latina serían reducidos a un "local tokenism” mediante el cual en América Latina sólo se puede hablar de sí misma, representarse a sí misma y satisfacer las exigencias de "autenticidad" disciplinaria mientras que el mundo se piensa en otros lugares, en Alemania, Inglaterra o Estados Unidos.

Las anteriores son algunas de las cuestiones institucionales que deberán discutirse y analizarse. En cuanto a los proyectos intelectuales no hay, por cierto, una relación uno a uno entre proyectos intelectuales y espacio institucional llamado "estudios culturales". Algunos de los debates y las inquinas que se generaron en los últimos años entre intelectuales residentes en América Latina y latinoamericanos residentes en Estados Unidos se debieron y deben, a mi entender, a la confusión entre proyectos intelectuales y espacios institucionales. Por ejemplo, cuando desde América Latina se dice que los “estudios culturales” son proyectos del Primer Mundo o de Estados Unidos, o imperialistas o como se quiera, se asume que junto con el nombre llegan también los proyectos intelectuales. Esto es, que al aceptar el nombre es necesario también aceptar los proyectos intelectuales. Las cosas pueden sin duda ser así, pero no tienen por qué serlo. Si el logo "estudios culturales" es hoy por hoy un logo conveniente que permite, como dije al principio, convencer a los decanos y rectores y, por otro lado, abrir espacios que no permiten abrir las disciplinas tradicionales no porque sean tradicionales sino porque están controladas por investigadores tradicionales y sobre todo conservadores, ese logo no tiene por qué llenarse con el proyecto intelectual de la Inglaterra de los 60 o con el de los estudios culturales “latinoamericanos” en Estados Unidos. Es más, puede ocurrir que los destellos del nombre no dejan ver que a su lado se están adelantando proyectos intelectuales innovadores que quedan tapados por la disputa institucional sobre el qué y el porqué de un nombre. ${ }^{7}$ Al separar espacio institucional de proyectos culturales es imperativo volver

\footnotetext{
${ }^{7}$ En Estados Unidos, por ejemplo, se han estado realizando proyectos innovadores en la American Philosophical Association, en el sector de filosofía no-noratlántica. Eduardo Mendieta ha sido y sigue siendo una de las figuras más prolíficas en ese sector. Ver su Latin America and postmodernity: a comtemporary reader, co-editado con Pedro Lange-Churión (2001); Teorías sin disciplina,
} 
a) sobre la historia de América Latina, desde la colonización del siglo xvi hasta el Plan Colombia y el ALCA y b) sobre las genealogías del pensamiento y del pensamiento crítico en América Latina en conexión con Africa y Asia; esto es, con aquellos espacios marcados por la historia del colonialismo en el mundo moderno. Es obvio que no se puede ignorar a Europa. El imperialismo no lo fue ni lo sigue siendo en términos económicos, políticos, legales e institucionales, sino también intelectuales. Europa y Estados Unidos “exportaron” el paquete completo, las formas de opresión y los proyectos críticos de oposición.

De modo que, en última instancia, el problema fundamental para mí no es tanto el de si los estudios culturales o los estudios poscoloniales o los estudios étnicos o de género son unos preferibles a los otros, el problema fundamental está en otra parte. Diría, para terminar, que la cuestión es más general y que puede plantearse en el nivel de las Humanidades, teniendo en cuenta, por cierto, la distribución actual del saber entre Humanidades, Ciencias Sociales y Ciencias Naturales. La tradición europea distingue entre Ciencias Humanas y Naturales y en las primeras incluye las ciencias sociales y las humanidades. Sin embargo sabemos que, a partir de la guerra fría, ciencias sociales como la economía, las ciencias políticas y la sociología se convirtieron en ciencias humanas “duras”, si se quiere, modeladas sobre las ciencias naturales. Pero no sólo eso, sino que pasaron de la necesidad de explicar al deseo de administrar la sociedad. En este sentido, a las Humanidades les corresponde el papel crítico que Immanuel Kant le había otorgado a la filosofía. Pienso que, hoy por hoy, frente a la globalización, al Plan Colombia y a la deuda externa en América Latina y, últimamente, a la rearticulación de la crisis, se imponen cuatro preguntas que podrán guiar varios proyectos intelectuales futuros:

— ¿Cuáles son los problemas y asuntos que exigen nuestra atención, que nos llaman, que nos invaden el cuerpo?

—¿Qué tipo de conocimiento/comprensión nos exige la historia, la sociedad y las genealogías intelectuales en las que elegimos inscribirnos?

—¿Desde qué perspectiva (disciplinaria, étnica, genérica, sexual, nacional, etc.) produciremos tal conocimiento o comprensión? Esta pregunta asume, por cierto, que la perspectiva disciplinaria no es neutra y que está marcada por el color, el género, la sexualidad, la nacionalidad (esto es, la lengua en la que se escribe y las genealogías inscriptas en esa lengua).

—¿Con qué fin? ¿Produciremos conocimientos y trataremos de comprender para “avanzar el conocimiento", para "llegar a la verdad” o para incidir en la transformación social y, en consecuencia, el conocimiento-comprensión producido estará en relación con los problemas y asuntos que nos exige la historia, la sociedad y las genealogías intelectuales en las que elegimos inscribirnos?

Estas preguntas son para mí fundamentales. La presentación de Edgardo Lander (en su presentación en Quito, titulada “La colonialidad del saber”) enfatizó el “color, el género y la geopolítica” de la epistemología moderna. Es decir, que la epistemología de la modernidad europea presupone un sujeto caracterizado por su masculinidad, por su

Latinoamericanismo, poscolonialidad y globalización en debate, co-editado con Santiago CastroGómez. Thinking from the underside of history: Enrique Dussel's philosophy of liberation, coeditado con Linda Martín Alcoff . 
blancura y por su europeidad. A ello yo agregaría, un sujeto que piensa y escribe en escritura alfabética y cuyas lenguas matrices son el griego, el latín y las lenguas “modernas” del conocimiento: el inglés, el francés y el alemán. Se me ocurre pensar que los "estudios culturales” no se han zafado todavía de esta prisión epistemológica. Han enfatizado la interdisciplina, pero manteniendo inconscientemente los principios epistemológicos comunes a todas las disciplinas modernas, desde las ciencias físicas y naturales, a las sociales y a las humanidades. Estos son, a mi entender, algunos de los problemas fundamentales a los que deberíamos dedicarnos a pensar críticamente sin olvidar, aunque a otro nivel, la necesidad del pensar estratégico de cómo usar los Estudios Culturales en diversos lugares institucionales, esto es, en las universidades del primer o del tercer mundo, o si cupiera, qué otros lugares que pueden existir fuera de las universidades donde el pensamiento crítico pueda continuar ejerciéndose. En última instancia, los proyectos intelectuales asociados con los Estudios Culturales en América Latina deberán, al menos durante las próximas décadas, partir de la colonialidad del poder como denuncia y crítica al racismo epistémico de la modernidad.

El espacio institucional es importante, claro, pero subsidiario en relación a los proyectos intelectuales. No es un fin, sino un medio. El problema se presenta cuando invertimos los términos y pensamos que los “estudios culturales” son un fin y no un medio. Los acontecimientos y procesos sociales, económicos, civiles, militares, psicológicos que se acentuaron a partir del 11 de septiembre creo que ponen a las claras que los problemas no están en cómo dividimos la torta disciplinaria sino en cuáles son los problemas de vida y muerte, de opresión y control estatal, de reacción y descontrol privado a los que todas las áreas del saber tienen, tenemos, la responsabilidad ética y política de contribuir a solucionar. El 11 de septiembre acentuó los límites del academismo y la necesidad de producción de conocimientos que contribuyan a transformar la comprensión hegemónica de la historia, de la colonialidad, del poder, del racismo imbricado en fantasmas de color de piel y de creencias religiosas. En fin, que contribuyan a comprender la cara del nuevo dios, de la nueva trinidad (capitalismo, militarismo y política) ante la cual sus defensores ofrecen sacrificios humanos, como entre los antiguos aztecas, y sus detractores no dudan en responder con ceguera semejante. La lista de preguntas que acabo de enumerar fueron enunciadas antes del 11 de septiembre. Después de esta fecha las preguntas no han perdido su razón de ser. Al contrario, se han hecho, para mí, más acuciantes.

BiBLIOGRAFÍA

Castro-Gómez, Santiago. La reestructuración de las ciencias sociales en América Latina. Bogotá: Pensar, 2000.

y Eduardo Mendieta. Teorías sin disciplina, latinoamericanismo, poscolonialidad y globalización en debate. México: Porruá; University of San Francisco, 1998.

Chen, Kuan-Hsing. "Voices from the outside: towards a new international localism". Cultural Studies 6/3 (1992): 476-84.

Dussel, Enrique. El último Marx (1863-1882) y la liberación latinoamericana: un comentario a la tercera y la cuarta redacción de "El capital”. Iztapalapa: Siglo Veintiuno Editores, 1990. 
Dworkin, Dennis. Cultural Marxism in Postwar Britain. History, the New Left, and the Origins of Cultural Studies. Durham: Duke University Press, 1997.

Frankenberg, Ruth y Latina Mati. “Crosscurrents, Crosstalk: Race, Postcoloniality and the Politics of Location”. Cultural Studies 7/2 (1993): 292-310.

Grosfoguel, Ramón. “Development, Modernity, and Dependency Theory in Latin America”. Nepantla. Views from South 1/2 (2000): 347-76.

Iander, Edgardo. La colonialidad del saber: eurocentrismo y ciencias sociales: perspectivas latinoamericanas. Caracas: FACES-UCV-UNESCO, 2000.

Mato, Daniel. "Estudios y otras prácticas latinoamericanas en cultura y poder”. Revista Venezolana de Economía y Ciencias Sociales 7/3 (2001): 85-112.

Mendieta, Eduardo y Linda Alcoff. Thinking from the Underside of History: Enrique Dussel's Philosophy of Liberation. Lanham, MD: Rowman \& Littlefield Publishers, 2000.

y Lange-Churión, Pedro. Latin America and Postmodernity: A Contemporary Reader. Amherst, NY: Humanity Books, 2001.

Mignolo, Walter. "Capitalism and Geopolitics of Knowledge: Latin American Social Thought and Latino/a American Studies”. Rethinking Area Studies. Juan Poblete, ed. Minneapolis: University of Minnesota Press, 2002.

"Descolonización epistémica y ética: la contribución de Xavier Albó y Silvia Rivera Cusicanqui a la reestructuración de las ciencias sociales desde los Andes”. Revista Venezoloana de Economía y Ciencias Sociales 7/3 (2001): 85-112.

Morley, Davis y Kuan-Hsing Chen. Stuart Hall. Critical Dialogues in Cultural Studies. London: Routledge, 1996.

Nelson, Cary y Lawrence Grossberg (Eds.). Marxism and the Interpretation of Culture. Urbana: University of Illinois Press, 1988.

Reynoso, Carlos. Apogeo y decadencia de los estudios culturales: una visión antropológica. Barcelona: Gedisa Editorial, 2000.

Rodríguez, Ileana. “Introduction”. The Latin American Subaltern Studies Reader. Durham: Duke University Press, 2001. 